\section{DE COMPLEMENTO A MOTOR: LA TRANSFORMACIÓN DE LA FUNCIÓN DEL OCIO Y TURISMO EN LAS ESTRATEGIAS DE DESARROLLO LOCAL. EL CASO DE LA RECUPERACIÓN Y VALORIZACIÓN DEL PATRIMONIO CULTURAL}

FROM COMPLEMENT TO MOTOR: THE CHANGING ROLE OF LEISURE AND TOURISM IN LOCAL DEVELOPMENT STRATEGIES. THE CASE OF THE RECOVERY AND VALORISATION OF CULTURAL HERITAGE

\author{
Joan Noguera Tur \\ Adrián Ferrándis Martínez \\ Mar Riera Spiegelhalder \\ Instituto de Desarrollo Local \\ Universitat de València \\ Joan.noguera@uv.es
}

\begin{abstract}
In the Fordist society, the productive activity focused development strategies, considering the role of leisure and tourism as "complementary" productive activities. The consolidation of post-productivism since the decade of 1990s has caused profound changes in individual and collective values and priorities. These have seen the introduction of a radically different conception about the role of leisure and tourism in the vital programming and, consequently, in the strategies of companies, organisations and governments. This paper provides a discussion of the principles of local development and the, more and more, central function of leisure and tourism in regional development strategies, through the presentation of a research methodology to assess the situation and potential of cultural resources in the configuration of endogenous local development strategies.
\end{abstract}

KEY WORDS: Leisure and tourism; local development; strategic planning.

\section{INTRODUCCIÓN}

Las múltiples formas de entender y disfrutar el ocio han ido evolucionando con el desarrollo, consolidación y transformaciones del estado de bienestar, así como con las disyuntivas a las que deben enfrentarse las personas a la hora de
RESUMEN: En la sociedad fordista, la actividad productiva centró las estrategias de desarrollo de los territorios dejando en segundo término, en el mejor de los casos, la función del ocio y del turismo como "complementos" de las actividades productivas "principales". La consolidación de la sociedad post-productivista desde la última década del pasado siglo ha supuesto profundas transformaciones en los valores y prioridades individuales y colectivas. Estos cambios han conllevado la implantación de una concepción radicalmente diferente respecto de la función del ocio y del turismo en la programación vital $y_{1}$ en consecuencia, en las estrategias institucionales y territoriales. El presente artículo aporta una discusión sobre los principios del desarrollo desde el territorio (local) y la función, cada vez más central, que tienen el ocio y el turismo en las estrategias de desarrollo territorial, a través de la presentación de una metodología y de los resultados de una investigación sobre la importancia de los recursos culturales en la configuración de estrategias endógenas de desarrollo local.

PALABRAS CLAVE: Ocio y turismo; desarrollo local; planificación estratégica.

elegir entre ocio y trabajo. Las decisiones individuales entre tiempo de trabajo y de ocio en la sociedad capitalista están asociadas a un conjunto de condicionantes que incluyen desde las circunstancias y "obligaciones" personales del individuo (disponibilidad de recursos económicos, gastos fijos, obligaciones contraídas, etc.) hasta las circunstancias 
derivadas de la coyuntura económica y social de una época determinada. En principio, puede tomarse como norma general que una persona estará dispuesta a trabajar más tiempo en la medida en que su retribución salarial aumente. Sin embargo, llegados a un nivel de ingresos determinado, la variación de horas deseadas de trabajo ante un cambio de renta mantiene el salario constante, mientras que la variación en horas deseadas de trabajo derivadas de un cambio de salario mantiene la renta constante (Case and Fair, 1999). Esto significa que, a partir de determinado nivel de renta, si las condiciones legales del mercado de trabajo lo permiten, el individuo no estará dispuesto a trabajar más en detrimento de su tiempo de ocio. Éste es el punto de partida que explica la instauración de una jornada de trabajo limitada por ley a un número de horas diarias y semanales $y$, al mismo tiempo, la consolidación de un tiempo de descanso anual retribuido para los trabajadores. Si añadimos a estos criterios las implicaciones de la revolución de los transportes y las comunicaciones, tenemos los ingredientes necesarios para la emergencia de una cultura del ocio o, cuanto menos, una cultura en la que el ocio desempeña una función relevante en la configuración de las aspiraciones vitales y en la organización de los individuos, las comunidades y el conjunto de la sociedad.

Aunque durante algunas décadas productivismo y cultura del ocio convivieron en direcciones evolutivas opuestas o, cuanto menos divergentes, en la sociedad fordista la actividad productiva centraba las estrategias de desarrollo de los territorios, dejando en un segundo término, en el mejor de los casos, la función del ocio y del turismo como "complementos" de las actividades productivas "principales" (Valls, 2004).

La consolidación de la sociedad post-productivista desde la última década del pasado siglo ha supuesto profundas transformaciones en los valores y prioridades individuales y colectivas. A través de estos cambios se ha producido la implantación de una concepción radicalmente diferente respecto de la función del ocio y del turismo en la programación vital $y_{1}$ en consecuencia, en las estrategias institucionales y territoriales (Noguera, 2009). El presente artículo aporta una discusión sobre los principios del desarrollo desde el territorio (local) y la función, cada vez más central, que tienen el ocio y el turismo en las estrategias de desarrollo territorial, a través de la presentación de una metodología para la recuperación y puesta en valor del patrimonio cultural como recurso para el desarrollo, entendiendo que el turismo, como factor fundamental de la cultura del ocio, se ha convertido, en las últimas dos décadas, en elemento central de las estrategias de desarrollo de muchos territorios, y que el patrimonio cultural contiene fortalezas y oportunidades que pueden aprovecharse para impulsar la economía, el empleo y la mejora social, mediante procesos impulsados desde las propias comunidades locales (Figuerola, 2006).

\section{El marco conceptual de la cultura como FACTOR DE DESARROLLO TERRITORIAL}

\subsection{La estrecha relación entre cultura y desarrollo}

Etimológicamente, el concepto de "cultura" hace referencia al "cultivo" de un individuo. Después de dos siglos en los que ha dominado una concepción cognitiva del concepto, ahora se recupera su significado inicial dinámico (Commission of the European Communities, 2010). Según qué perspectiva tomemos, la cultura puede ser entendida "como el sistema compartido de creencias, valores y prácticas que definen un conjunto humano" (Rausell et al., 2007, p. 46), pero también "cultura es más entendida como aquel conjunto de significados, prácticas y expresiones compartidas de un colectivo social" (Rausell, 2006, p. 3). Así, la cultura se convierte, al mismo tiempo, en un derecho cultural que forma parte intrínseca de la condición humana, tal como reconocen la Declaración de Derechos Humanos de 1948 y la Agenda 21 de la Cultura. Este último documento, referencia del enfoque estratégico que guía la política cultural europea, clarifica la comprensión del concepto de "cultura" como un proceso dinámico y capaz de generar libertad. Este enfoque entronca de manera clara con la cultura como instrumento para la generación de valores. En este sentido, algunos autores hablan del derecho a la cultura como parte fundamental de los derechos de las personas. Así, Delgado (2001, p. 49) afirma que: "las políticas culturales guiadas por valores toman en consideración que la cultura es un derecho humano, arraigado en la parte más profunda de la dignidad". El derecho a la cultura queda reconocido por la mayoría de los textos constitucionales de paises occidentales, en el caso del Estado español en su artículo 149, que la establece como un deber y una atribución esencial del Estado. 
Es a partir de esta concepción de la cultura como derecho, que toma sentido hablar de políticas culturales, en tanto que todos los individuos de una comunidad pueden ser partícipes de la vida cultural; es decir, se reconoce su derecho a hacerlo. En este contexto, el sector público tiende a adoptar un papel destacado: "si la libertad y el desarrollo implican a la cultura, las instituciones públicas necesitan encontrar leyes y políticas culturales, y después programas y proyectos, que puedan garantizar que todos los ciudadanos/habitantes de la ciudad puedan llegar, con y a través de la cultura, a un desarrollo humano más completo" (Pascual y Dragojevic, 2007, p.13). La incorporación de la cultura como derecho y como generador de valores permite ampliar el clásico "triángulo de desarrollo" basado en la economía, el medio ambiente y la inclusión social, que adopta asi la forma de un "cuadrado de desarrollo" (Figura 1). En este modelo actualizado, las políticas, programas y acciones son evaluadas según el impacto que pueden producir en la esfera económica, medioambiental, de inclusión social y cultural (Pascual y Dragojevic, 2007).

FIGURA 1. EL NUEVO CUADRADO DEL DESARROLLO

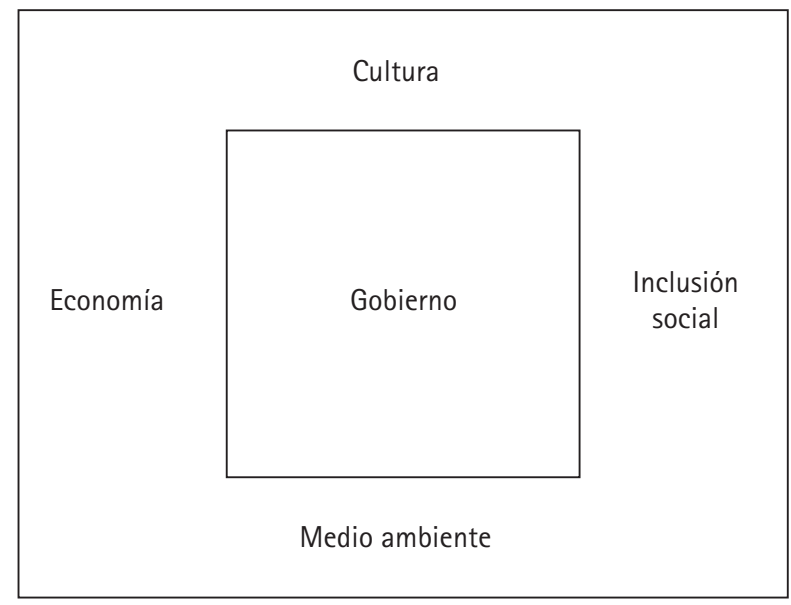

Fuente: Pascual et al. (2007).

De acuerdo con esta concepción del desarrollo, la política cultural se aleja de una concepción superficial y superflua para convertirse en una herramienta de primer orden que puede contribuir a la consolidación de valores individuales y colectivos, a mejorar la cohesión social de una comunidad $y$, en consecuencia, su capital social y relacional
(Moragues, 2006). Así, la política cultural constituye un instrumento fundamental para el desarrollo, al favorecer la localización de actividades productivas, la generación de capital social y un sentido de pertenencia de la población a su comunidad y territorio (Figura 2).

FIGURA 2. CULTURA Y TERRITORIO: UNA RELACIÓN PARA EL DESARROLLO

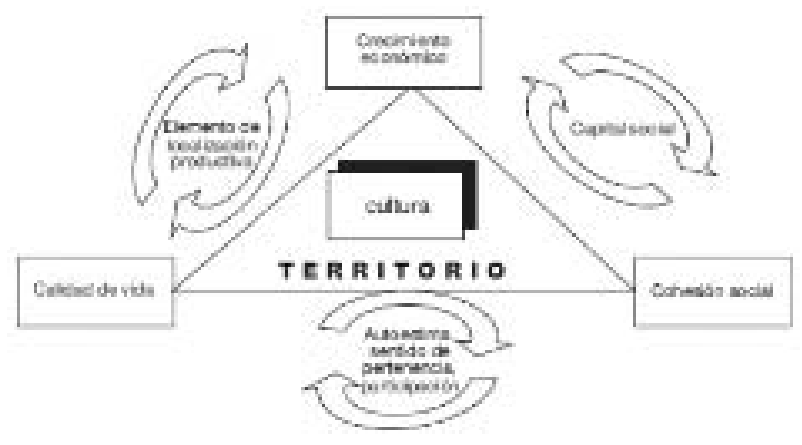

Fuente: Rausell et al. (2007).

\subsection{Desarrollo local y estrategias basadas en la recuperación y puesta en valor de los recursos culturales}

A inicios de la segunda década del siglo XXI, dos procesos de largo recorrido $y$, con frecuencia, contradictorios, vienen condicionando el modo en que se lleva a cabo la relación entre cultura y desarrollo. Por un lado, la tendencia de la política neoliberal a privatizar y comercializar funciones que tradicionalmente habían sido desarrolladas desde el ámbito público, ha afectado sustancialmente la forma en que los gobiernos se involucran en las industrias culturales, y ha provocado la redefinición de las funciones de los actores involucrados a la hora de proveer políticas culturales (Elliot, 1997; Hall, 2000; Pearce, 1992). Por otro lado, el tránsito desde un modelo en el que predominaba la regulación centralizada a otro más orientado a la gobernanza descentralizada, favorece la implantación de políticas y estrategias de desarrollo más ajustadas a las necesidades reales de cada territorio y sociedad local (Pike et al., 2011). Por esta razón, la política cultural queda supeditada a las prioridades y necesidades de los territorios 0 , al menos, de las instituciones que los gobiernan. Ello

ARBOR Vol. 188754 marzo-abril [2012] 379-393 ISSN: 0210-1963 
tiene importantes implicaciones tanto positivas (mejor ajuste a la realidad social y territorial, etc.), como negativas (perspectiva potencialmente miope o excesivamente localista, desajustes con las estrategias y políticas culturales vecinas o generales, etc.). Algunos autores aportan argumentos teóricos a favor de este enfoque "local" de la política cultural. Así, Nogués (2007) señala que la cultura constituye un compendio de manifestaciones, circunstancias y contextos que adquieren sentido dentro de un grupo específico y dan sentido a la vida social. De este modo, cualquier proceso de desarrollo planificado puede ser sentido como propio, solo si perduran en él la memoria histórica y cultural que dan sentido a la cotidianidad y a la apropiación del territorio por parte de sus habitantes (Nogués, 2007).

En el marco de estos dos procesos, la cultura ha pasado a ocupar un lugar central en el diseño de las estrategias de desarrollo, por su capacidad para generar economía y empleo, para incrementar el atractivo turístico y para reforzar el sentido de pertenencia. En este sentido, la Organización Mundial del Turismo (2000) considera la cultura como un factor determinante del crecimiento del consumo turístico. De igual modo, otros organismos internacionales y las políticas de los países más desarrollados, otorgan una creciente importancia a la recuperación y puesta en valor de los recursos culturales por sus múltiples beneficios para el desarrollo económico, el refuerzo de la identidad y la cohesión social, entre otros. La relevancia del patrimonio cultural se manifiesta también a nivel comunitario. El Libro Verde de las Industrias Culturales y Creativas (COM, 2010), especifica que "los sectores culturales y creativos fomentan la creatividad y contribuyen a la innovación en otros sectores de la economía. [...] También actúan como multiplicador del desarrollo local ya que constituyen un poderoso catalizador para atraer turistas, resultan de una importancia estratégica para el crecimiento y la creación de empleo en las ciudades, y tienen impactos sociales significativos a nivel local..." (COM, 2010, p.3). Estos y otros ejemplos ilustran la creciente importancia dada desde las instituciones al factor cultural en los procesos y estrategias de desarrollo.

De acuerdo con estas argumentaciones, la política cultural puede constituir una herramienta para la mejora de la cohesión social de una comunidad y, por tanto, de su capital social y relacional, convirtiéndose así en una herramienta fundamental para el desarrollo. Las sociedades cohesionadas resultan más fáciles de gestionar, con relaciones individuales más densas y menos conflictivas. Por extensión, el capital relacional (las instituciones, relaciones y normas que configuran las interacciones sociales) hará posible un mayor dinamismo económico, incrementando, a su vez, la calidad de vida del conjunto de la sociedad.

Sin embargo, para que las políticas culturales sean eficientes e influyentes, es necesario que se inserten adecuadamente en la realidad local. Para ello, el enfoque endógeno y participativo facilita una toma de decisiones participadas no solo por las instituciones públicas sino, además, asesorada, elaborada, implementada e, incluso, evaluada, por los demás actores involucrados en el proceso (Pascual y Dragojevic, 2007).

Las políticas culturales participativas emanan de una planificación estratégica que moviliza y sensibiliza a la ciudadanía. Para ello es esencial partir de un análisis de la situación inicial de los recursos culturales y de la función de la cultura en el proceso de desarrollo.

Uno de los principales retos a la hora de planificar la función de la cultura en el desarrollo del territorio está en identificar la variedad de recursos culturales existentes y valorar su relevancia. Hablar de bienes o recursos culturales, es hablar de bienes materiales e inmateriales, percibidos y valorados de forma diferente por los actores locales en función de sus intereses y posicionamientos. La valoración es, quizá, el proceso más complejo dado que está sujeto a un importante componente de subjetividad y de emocionalidad. Sin embargo, valorar la relevancia e importancia de los recursos culturales es un paso fundamental en el proceso de recuperación, puesta en valor e integración en la estrategia de desarrollo. En este sentido, algunos autores proponen una serie de argumentos en función de los que se pueden valorar los bienes patrimoniales y que comprenden: (i) el valor artístico atribuido al bien patrimonial, (ii) la singularidad, (iii) la antigüedad y el grado de conservación. La adecuada valoración de los bienes culturales permitirá, por un lado, preservarlos $y_{\text {, en }}$ algunos casos, recuperarlos. Pero también nos va a permitir identificar aquellos bienes que, en mayor o menor grado, pueden ser incorporados como recursos estratégicos para el desarrollo. 
Por tanto, si admitimos que la cultura contribuye al desarrollo territorial, que ésta se puede promover y manifestar a través de políticas culturales participativas y que, en consecuencia, los actores locales tienen una función central en el proceso de diseño, gestión y evaluación, resulta necesario proceder al inventario y catalogación de los bienes culturales de un territorio, como paso previo para su recuperación y puesta en valor. De este modo, conseguiremos que la cultura y los recursos culturales se constituyan en elementos de generación de identidad y de disfrute de la sociedad local, o bien en productos turísticos con atractivo de mercado.

Hasta el momento se ha tratado de establecer la relación conceptual entre cultura y desarrollo territorial, y se ha establecido la necesidad de incorporar la cultura como uno de los pilares fundamentales de los procesos de planificación del desarrollo. Desde esta perspectiva se procede, a continuación, a explicar un método para la recuperación y puesta en valor de los recursos culturales basado en los principios de gobernanza local, participación, estrategia y desarrollo sostenible.

\section{UN MÉTOdO PARA LA RECUPERACIÓN Y PUESTA EN VALOR DE LOS RECURSOS CULTURALES LOCALES DESDE EL ENFOQUE LOCAL DEL DESARROLLO}

\subsection{El marco territorial}

El método que se describe a continuación ha sido elaborado teóricamente por miembros del Instituto Interuniversitario de Desarrollo Local de la Universitat de València, y testado con éxito en el municipio de Tavernes de la Valldigna, localidad de aproximadamente 20.000 habitantes situada en la costa meridional del Golfo de Valencia. El poblamiento del municipio se concentra en el núcleo histórico, situado en el lateral izquierdo del Valle de La Valldigna, a $4 \mathrm{~km}$ de la línea de costa, y en el núcleo de la "playa", desarrollado en los años 70 de acuerdo con un modelo de turismo residencial altamente estacionalizado, que hoy muestra todas las debilidades de los destinos maduros de sol y playa (elevada estacionalidad, importantes carencias en cuanto a servicios y equipamientos, implantación y desarrollo al margen de procesos coherentes de planeamiento urbano, etc.).
El valle ha sido escenario de importantes asentamientos humanos desde la prehistoria y, todavía hoy, cuenta con un notable patrimonio en forma de cuevas con ejemplos de arte rupestre, la mayoría reiteradamente vandalizadas. El municipio cuenta también con muestras de interés arquitectónico y etnológico que incluyen desde edificios de carácter religioso repartidos por el núcleo histórico hasta algunas alquerías y molinos repartidos por el término, una torre-vigía o un castillo de la dominación musulmana. El patrimonio inmaterial no es menos importante y cuenta con elementos singulares de gastronomía local, una fuerte tradición asociativa musical, la festividad de las Fallas, y un abundante elenco literario de tradición oral y escrita, etc. Hasta ahora, la inmensa mayoría de este patrimonio no ha sido objeto de uso turístico, o bien ha tenido un uso limitado a las tradiciones y costumbres locales, sin mayor proyección. No ha existido, hasta la fecha, un análisis exhaustivo y riguroso de las posibilidades de recuperación y puesta en valor de los valores patrimoniales y culturales del municipio $y$, en consecuencia, no se ha establecido su relevancia, tanto por lo que respecta a las necesidades y demandas de la sociedad local, como para el impulso de procesos de desarrollo que incluyan la cultura como uno de sus ejes.

Durante el año 2009, el Ayuntamiento de Tavernes de la Valldigna, por medio de su alcalde, manifestó a la Universidad de Valencia su preocupación por el estado de abandono de buena parte del patrimonio cultural y natural del municipio, y por el escaso o nulo uso turístico del mismo. Tras este discurso estaba implícito el interés en llevar a cabo un análisis de los recursos culturales del municipio y de su potencial de recuperación y puesta en valor, tanto para su utilización como recurso de desarrollo, como para el uso y disfrute de la ciudadanía, y el reforzamiento de la identidad territorial. Éste fue el punto de partida para la puesta en marcha de un análisis estratégico de los recursos culturales del municipio.

\subsection{Un análisis estratégico para la recuperación y puesta en valor de los recursos culturales}

Como en todos los estudios e informes encargados por una institución local, los primeros pasos suelen referirse al modo de hacer operativa una idea que, con frecuencia, parte de la autoridad política (en este caso, el alcalde) pero que puede tener defensores o detractores

ARBOR Vol. 188754 marzo-abril [2012] 379-393 ISSN: 0210-1963

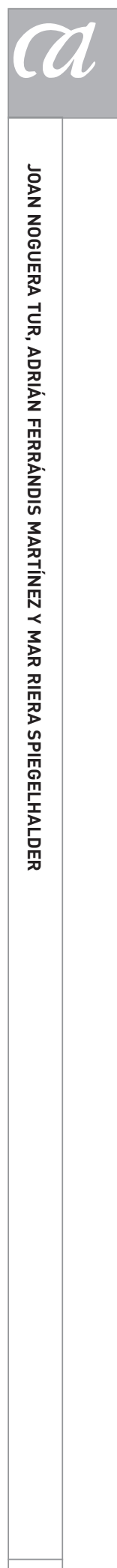

383 
en la propia institución. En muchos casos, además, el encargo es una idea más o menos abstracta de lo que se pretende conseguir, pero no se da mucha información añadida sobre el trabajo previo existente, el/los departamento(s) y personas en la administración local que pueden tener interés en el estudio, y las personas a las que debe acudirse como punto de referencia para la obtención de información, ya que por interés o porque han recibido el encargo de forma explícita, dedicarán tiempo y esfuerzo a resolver las necesidades y demandas del equipo técnico externo. Por tanto, las primeras semanas de trabajo suelen ser de "aterrizaje" en la realidad institucional local, y de "ubicación" en el marco de competencias, de búsqueda de aliados, y de identificación de opositores.

Una vez caracterizada la situación institucional de partida, el siguiente paso consiste en hacer lo propio con respecto al objeto de análisis; es decir, caracterizar la situación inicial de los recursos culturales del municipio. Para ello se requieren técnicas de inventario que permitan, mediante el diseño de fichas estandarizadas, recoger información sobre el estado de cada bien y recurso cultural. De este modo se llega a un Inventario y Catalogación de los Recursos Culturales. Este inventario debe acompañarse de un análisis sobre las redes y jerarquias de instituciones y organizaciones implicadas en el funcionamiento de cada recurso cultural.

El inventario constituye la "materia prima" que permitirá elaborar un diagnóstico acertado de la situación de los recursos culturales. En este caso, con una etapa intermedia, ya que nos interesa valorar la medida en que los bienes y recursos culturales pueden convertirse en elementos de uso turístico y de generación de identidad. Por ello, al inventario se añade un "Análisis de Factibilidad" en el que se valora el estado actual y potencial de futuro de cada recurso a partir de indicadores cualitativos ponderados. Con esta información se llega a un "diagnóstico de recursos culturales". Sin embargo, hasta ese momento, el diagnóstico es meramente técnico, fruto de los análisis llevados a cabo por el equipo de la Universidad. En el marco de un proceso de desarrollo endógeno y participativo cabe incorporar un mecanismo riguroso de participación ciudadana que permita contrastar los resultados técnicos y validarlos con los puntos de vista y conocimiento experiencial de la sociedad local.

\subsection{El proceso de inventario y catalogación de los recursos culturales}

Como se ha dicho, el primer paso en el proceso de análisis de la situación de los bienes y recursos culturales requirió de su identificación, localización cartográfica, inventario y catalogación, dando cabida tanto a los recursos materiales como a los inmateriales o simbólicos (festividades, eventos, tradición gastronómica, etc.). Con el fin de transformar la catalogación que proporciona el PGOU en un Inventario Valorativo de Recursos Culturales que incorporase las variables necesarias, se diseñó una Ficha de Inventario (Figura 3) con la que se visitaron y analizaron los recursos. Mediante este procedimiento se determinó la relevancia de cada recurso en función de aspectos como su estado de conservación, entorno, servicios existentes, potencial turístico, etc.

El inventario recogía, entre otra información, el tipo de recurso, la existencia de declaración oficial de protección, localización, coordenadas UTM, breve descripción, fotografía aérea de localización, imagen y la definición de usos actuales. En el caso de los recursos inmateriales o simbólicos se recogían, entre otros, la denominación, el tipo de recurso, la declaración oficial, las coordenadas UTM, el organismo responsable de su gestión/celebración, la periodicidad del acontecimiento, una foto aérea del emplazamiento, una imagen, una breve descripción y observaciones.

\subsection{El Análisis de Factibilidad de los Recursos Culturales}

A partir de la información de inventario, el Análisis de Factibilidad permite medir la dificultad para transformar un bien o recurso cultural en un producto turístico o puramente cultural. No se trata, por tanto, de convertir en recurso turístico mercantilizado todo el patrimonio cultural del municipio, sino que se plantea como finalidad igualmente válida preservar/recuperar un bien cultural como elemento de identidad.

El Análisis de Factibilidad utiliza modelos de categorización y jerarquización para determinar el valor de uso de los bienes y recursos culturales. Este tipo de análisis facilita la valoración objetivable de los recursos culturales a partir de un conjunto de variables discretas: (i) accesibilidad (externa al recurso, área territorial exterior, e interna, hasta el propio recurso); (ii) entorno; (iii) visibilidad del recurso 
FIGURA 3. EJEMPLO DE FICHA DE INVENTARIO

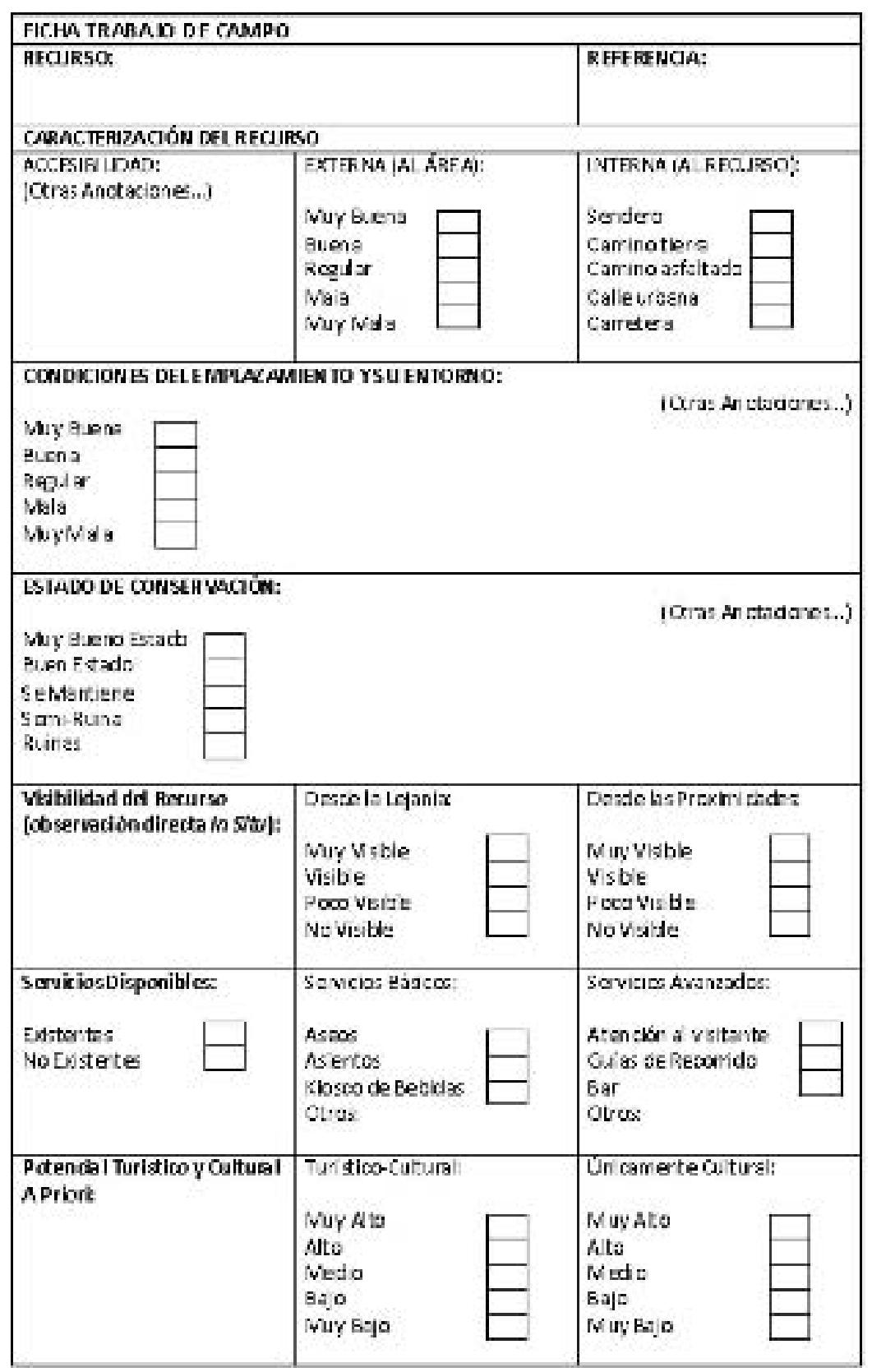

Fuente: Elaboración propia.

in situ; (iv) servicios disponibles; (v) protección legislativa; (vi) potencial turístico y cultural. En el caso de los recursos inmateriales, dada su singularidad, se utilizó otro conjunto de variables: (i) entorno; (ii) influencia en la economía local; (iii) generación de identidad cultural; (iv) trayectoria histórica y periodicidad; (v) implicación institucional; (vi) visibilidad; (vii) disfrute; (viii) protección legislativa; (ix) potencial turistico y cultural. 
Dado que cada variable estaba definida por un rango de valores diferente, resultó necesario homogeneizar todos los rangos. Para ello se procedió del siguiente modo:

Cada variable viene definida por un rango específico:

$$
X_{i}=\left[X_{i} \min , X_{i} \max \right]
$$

donde $X_{i}$ es el valor que adopta la variable; $X_{i}$ min es el valor mínimo que puede tener, $\mathrm{y}_{\mathrm{i}} \max$ el valor máximo. Los rangos iniciales de cada variable son diferentes. Por ello, es necesario homogeneizar todas las variables $X$, lo que significa que deseamos convertirlas en el intervalo:

$$
X_{n}=[\text { nnorm, }- \text { nnorm }]
$$

Definimos el rango de homogeneización como:

$$
X=[0,1]
$$

A continuación procedemos a transformar el rango $\left[X_{i} \min\right.$, $\left.X_{i} \max \right]$ en un rango desde 0 hasta otro valor $X$. Esto se consigue si al valor de $X_{i}$ le restamos $X_{i}$ min. Haciendo esta resta, $X_{i}$ min resultaría 0 , mientras que, en el caso de $X_{i} \max$, quedaría $X_{i} \max -X_{i} \min$. El rango queda así entre $\left[0, X_{i} \max -X_{i} \min \right]$ y $X_{n}$ :

$$
X_{n}=X_{i}-X_{i} \min ;
$$

Con estos valores homogéneos podemos transformar nuestro rango inicial en un rango entre 0 y 1 . Para ello, es necesario dividir $X_{n}$ entre $\left(X_{i} \max -X_{i} \min \right)$. Así, para un rango inicial $\left[0, X_{i} m a x-X_{i} m i n\right]$ dividimos ambos números entre $\left(X_{i} \max -X_{i} \min \right)$ y obtenemos $[0,1]$. De este modo, $X_{n}$ resulta:

$$
X_{n}=\left[\left(X_{i}-X_{i} \min \right) /\left(X_{i} \max -X_{i} \min \right)\right]
$$

Tras la aplicación de la fórmula anterior, las variables están disponibles para ser comparadas ya que todas comparten el mismo rango. Debe puntualizarse que en cuatro de las variables no se procedió del modo indicado, por tratarse de variables dicotómicas. Se trata de las variables "protección de la legislación" (en recursos materiales e inmateriales), "servicios disponibles" y "generación de identidad cultural". Dado que su rango viene definido por $X=[0,1]$, no fue necesario realizar ninguna transformación.
Una vez establecidos rangos homogéneos para todas las variables consideradas, se procedió a su ponderación, para reflejar su relevancia. A tal fin, se establecieron tres categorias. Cuando la variable se consideraba "muy relevante", se multiplicó su valor por 1,5. Para las variables consideradas "relevantes", se mantuvo su valor (multiplicación por 1). Finalmente, el valor de las variables consideradas "poco relevantes" se multiplicó por 0,5. En este caso, se utilizó la devaluación del valor y no el nulo 0 con el fin de evitar la anulación de la variable. Para ambos tipos de recursos, materiales e inmateriales o simbólicos, se siguió el mismo procedimiento en la ponderación, lo que permite una comparación más sencilla en caso de ser necesario.

Con el fin de legitimar el Análisis de Factibilidad se llevó a cabo un proceso de participación que permitió la incorporación de la opinión de los actores locales en el proceso de planificación. La metodología seguida se adaptó a la empleada por los Talleres de Visualización de Futuro (EASWEuropean Awareness Scenario Workshop) de la Dirección General XIII de la Comisión de la UE.

Tras las modificaciones pertinentes en el Análisis y Diagnóstico, se pasó al diseño de propuestas de actuación. Las propuestas se agrupan en líneas estratégicas, que a su vez se concretan en actuaciones, el desarrollo de cada una de las cuales responde a las siguientes preguntas: ¿de qué se trata? motivación (por qué), ¿quién lidera y quién participa?, prioridad, costes, ámbito de acción y fuentes de financiación.

\section{Resultados}

Los resultados del Análisis de Factibilidad (Figura 4) representan la puntuación total ponderada obtenida por cada uno de los recursos incluidos en el inventario. En este caso, la valoración global obtenida por cada recurso (incluyendo tanto el estado actual como el potencial de uso), puede alcanzar un máximo de 18,50 puntos. En esta valoración compuesta, el "estado actual" no implica mayor o menor importancia de los recursos en la estrategia de futuro, sino una estimación del estado actual del recurso en términos de conservación y uso. Por otro lado, el "potencial futuro" apunta a la relevancia del recurso en la estrategia cultural de futuro. En esta combinación ponderan con más fuerza los factores de estado y uso actual que los de potencial. 
FIGURA 4. VALOR TOTAL PONDERADO (MÁX. 18,5): RECURSOS MATERIALES

\begin{tabular}{|l|c|}
\hline RECURSOS MATERIALES & \\
\hline Recurso & Total ponderado \\
\hline
\end{tabular}

\begin{tabular}{|c|c|}
\hline Núcli Històric (S. XI- S.XV) & 16,17 \\
\hline Iglesia Parroquial San Pedro Apóstol & 15,04 \\
\hline Ermita del Santísimo Cristo del Calvario & 14,00 \\
\hline Ermita San Llorenç & 13,88 \\
\hline Iglesia Parroquial de San José & 13,33 \\
\hline Casa Consistorial & 12,67 \\
\hline Yacimiento El Rafol & 12,25 \\
\hline Yacimiento Abrics de Mossen Ricardo o Fontetes de Cantus & 11,67 \\
\hline Ullal de les Penyetes & 11,58 \\
\hline Yacimiento El Massalali & 11,42 \\
\hline Clot de la Font & 11,38 \\
\hline Castillo Alcalá de Alfandech o Els Castellets & 10,96 \\
\hline Pont del Vadell & 10,75 \\
\hline Yacimiento Serreta del Pas Buvap & 10,50 \\
\hline Torre de Guaita de la Valldigna & 10,17 \\
\hline Moli del Plà Vell & 10,04 \\
\hline Font del Canut & 9,88 \\
\hline Antiguo "Hospital de Sant Roc" & 9,42 \\
\hline Cova del Frare & 9,21 \\
\hline Cova del Caçador & 9,04 \\
\hline Ullal Gran & 8,96 \\
\hline Yacimiento El Plà & 8,92 \\
\hline Yacimiento Moleta de Rafol & 8,88 \\
\hline Yacimiento Penya del Mig dia & 8,88 \\
\hline Yacimiento L'Umbria & 8,83 \\
\hline Yacimiento Racó del Cementeri & 8,79 \\
\hline Barranco Bolomor & 8,75 \\
\hline Sendas & 7,96 \\
\hline Yacimiento El Teuleret & 7,92 \\
\hline Assut de Tavernes & 7,17 \\
\hline Moli de Tonet & 6,54 \\
\hline Yacimiento L'Alcudiola & 6,50 \\
\hline Cordón dunar & 6,13 \\
\hline El Molló & 5,88 \\
\hline Yacimiento Muntanya de Rafel & 5,50 \\
\hline
\end{tabular}

Fuente: Elaboración propia. 
En el caso de los recursos inmateriales, el valor de la puntuación máxima ponderada se establece en 10. Al igual que en el caso anterior, En esta valoración compuesta, el "estado actual" no implica mayor o menor importancia de los recursos en la estrategia de futuro, sino una estimación del estado actual del recurso en términos de conservación y uso. Por otro lado, el "potencial futuro" apunta a la relevancia del recurso en la estrategia cultural de futuro. En este caso, la valoración global señala a aquellos recursos mejor arraigados o de mayor proyección.

FIGURA 5. VALOR TOTAL PONDERADO (MÁX. 10,0): RECURSOS INMATERIALES

\begin{tabular}{|l|r|}
\hline Recurso & Total Ponderado \\
\hline
\end{tabular}

\begin{tabular}{|c|c|}
\hline Festival Sete Sois Sete Luas & 7,45 \\
\hline Dia de la Valldigna & 7,025 \\
\hline Dia de la "Sang" & 7,025 \\
\hline Trobada Internacional de Dramaturgia & 6,9 \\
\hline Festes Patronals en honor al Santissimo Crist de la sang i la Divina Aurora & 6,15 \\
\hline Llegenda Bolomor & 6,13 \\
\hline Santa Cecilia & 5,70 \\
\hline Festival Pop al Carrer & 5,525 \\
\hline Festivitat de Sant Antoni Abad & 5,53 \\
\hline Olles & 5,38 \\
\hline Fira de Productes Valencians (Sant Jaume) & 5,325 \\
\hline Tradició Arrosera & 5,125 \\
\hline Falles de Tavernes de la Valldigna & 5,03 \\
\hline Setmana Santa de Tavernes de la Valldigna & 5,03 \\
\hline Postres & 5,00 \\
\hline Programació Teatral Estable & 4,95 \\
\hline Dia de la Comunitat Valenciana & 4,90 \\
\hline Coques Mestall & 4,75 \\
\hline Pilota valenciana & 4,75 \\
\hline Tradició Dansa i Música & 4,13 \\
\hline Sant Llorenç & 4,03 \\
\hline Actuacions Estivales & 4,03 \\
\hline Salsamorra & 4,00 \\
\hline Sant Joan & 3,90 \\
\hline Concentració Motera & 3,40 \\
\hline Carnestoltes & 2,90 \\
\hline Primer de Maig & 2,9 \\
\hline Cicle Concerts Cámera & 2,85 \\
\hline Mercat & 2,38 \\
\hline Campionat Seven Rugbi & 1,65 \\
\hline Carns i Embotits & 1,25 \\
\hline
\end{tabular}

Fuente: Elaboración propia. 
Una visión más detallada, centrada únicamente en los resultados del análisis de potencial de uso turístico y de conservación cultural, permite separar la valoración del estado actual de la valoración del potencial de uso, para así identificar los elementos del patrimonio cultural con mayor proyección con vistas al diseño de la estrategia turístico-cultural. La valoración del potencial se realizó diferenciando el potencial turístico-cultural y el potencial únicamente cultural. La Figura 6 muestra el ejemplo de este análisis para el caso de los recursos materiales.

FIGURA 6. ANÁLISIS DE FACTIBILIDAD DE LOS RECURSOS CULTURALES (AFRC). POTENCIAL TURÍSTICO-CULTURAL DE LOS RECURSOS MATERIALES

\begin{tabular}{|l|l|l|l|}
\hline \multirow{2}{*}{ Recurso } & \multicolumn{2}{|c|}{ Potencial Turistico y Cultural } & \multirow{2}{*}{ Potencial total (sobre 2) } \\
\cline { 2 - 3 } & Turistico-Cultural & Únicamente cultural & \\
\hline
\end{tabular}

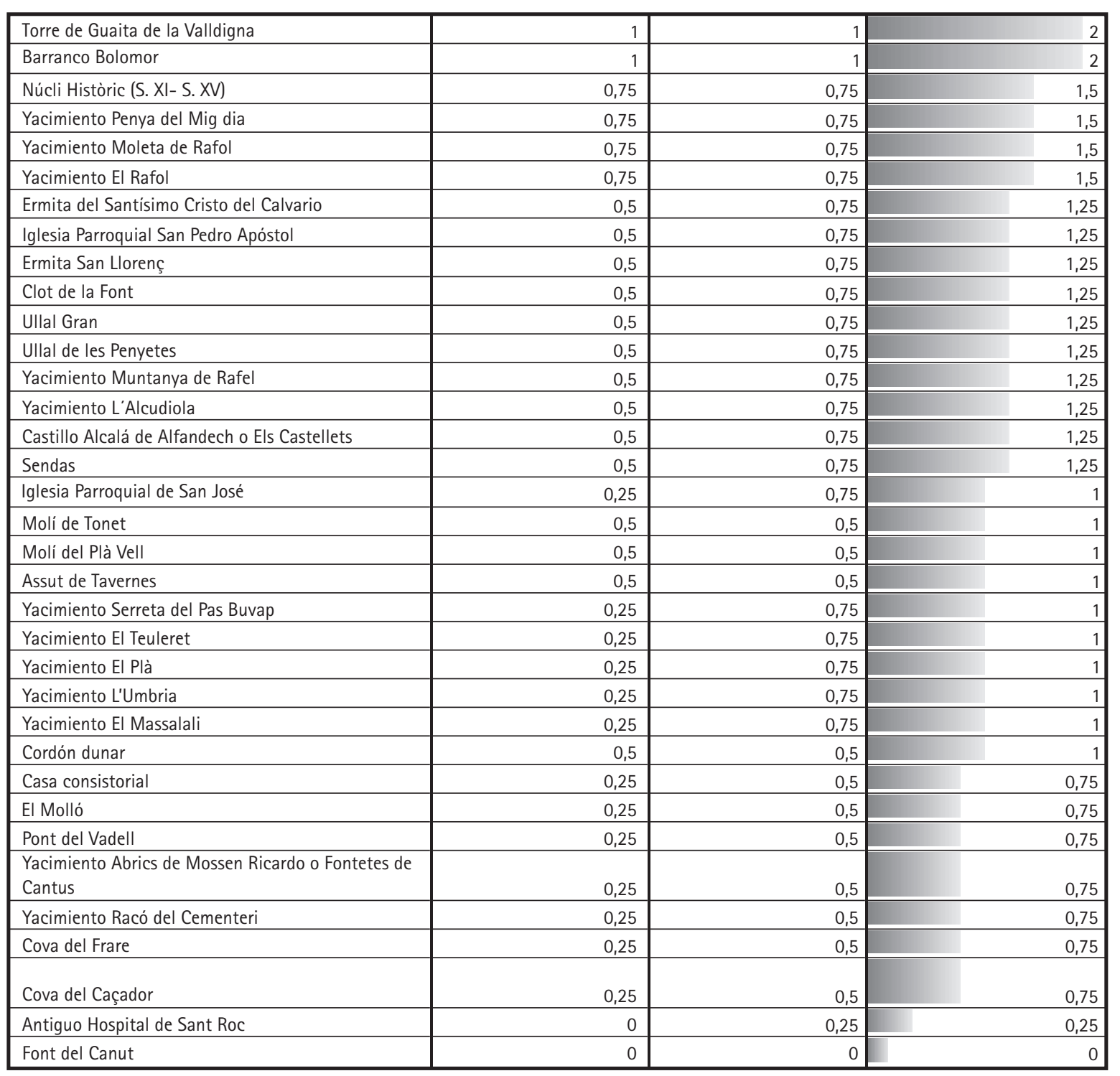

Fuente: Elaboración propia. 
El último elemento del Análisis de Factibilidad proporciona, para cada recurso considerado, la comparación entre la puntuación correspondiente al estado actual del recurso y su potencial de uso. Este ejercicio posibilitó la determinación de los elementos del patrimonio material e inmaterial que tienen una mejor valoración en relación a su estado actual $y_{\text {, en cambio, bajas }}$ expectativas de uso potencial, o viceversa. Asi, se evidencian los elementos sobre los que ha de pivotar la estrategia de futuro.

FIGURA 7. ANÁLISIS DE FACTIBILIDAD DE LOS RECURSOS CULTURALES (AFRC).

COMPARACIÓN ENTRE EL ESTADO DE CONSERVACIÓN Y EL POTENCIAL DE USO: RECURSOS INMATERIALES

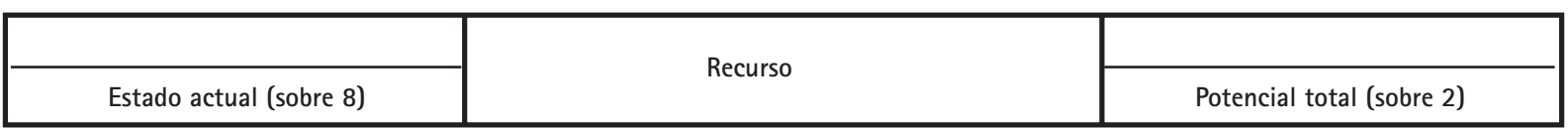

\begin{tabular}{|c|c|c|}
\hline 7,45 & Festival Sete Sois Sete Luas & 1,75 \\
\hline 6,9 & Trobada Internacional de Dramaturgia & 1,75 \\
\hline 5,525 & Festival Pop al Carrer & 1,5 \\
\hline 4,95 & Programació Teatral Estable & 1,5 \\
\hline 7,025 & Dia de la Valldigna & 1,25 \\
\hline 5,03 & Falles de Tavernes de la Valldigna & 1,25 \\
\hline 5,03 & Setmana Santa de Tavernes de la Valldigna & 1,25 \\
\hline 7,025 & Dia de la "Sang" & 1,25 \\
\hline 5,125 & Tradició Arrosera & 1,25 \\
\hline 5,38 & Olles & 1,25 \\
\hline 6,13 & Llegenda Bolomor & 1,25 \\
\hline 6,15 & $\begin{array}{l}\text { Festes Patronals en honor al Santíssimo Crist de la } \\
\text { sang i la Divina Aurora }\end{array}$ & 1 \\
\hline 4,75 & Coques Mestall & 1 \\
\hline 5,00 & Postres & 1 \\
\hline 5,53 & Festivitat de Sant Antoni Abad & 0,75 \\
\hline 3,90 & Sant Joan & 0,75 \\
\hline 4,90 & Dia de la Comunitat Valenciana & 0,75 \\
\hline 5,70 & Santa Cecilia & 0,75 \\
\hline 5,325 & Fira de Productes Valencians (Sant Jaume) & 0,75 \\
\hline 4,03 & Actuacions Estivales & 0,75 \\
\hline 3,40 & Concentració Motera & 0,75 \\
\hline 2,85 & Cicle Concerts Cámera & 0,5 \\
\hline 4,00 & Salsamorra & 0,5 \\
\hline 4,75 & Pilota valenciana & 0,5 \\
\hline 2,90 & Carnestoltes & 0,25 \\
\hline 2,9 & Primer de Maig & 0,25 \\
\hline 4,03 & Sant Llorenç & 0,25 \\
\hline 1,65 & Campionat Seven Rugbi & 0,25 \\
\hline 4,13 & Tradició Dansa i Música & 0,25 \\
\hline 2,38 & Mercat & 0 \\
\hline 1,25 & Carns i Embotits & 0 \\
\hline
\end{tabular}

Fuente: Elaboración propia. 
Durante el proceso de participación ciudadana se presentaron los resultados obtenidos en las etapas previas. Mediante una técnica de trabajo en grupos se recabaron opiniones, comentarios y propuestas directas de los ciudadanos. Aquellas que fueron apoyadas por la mayoria se recogieron en las líneas estratégicas, al tiempo que fueron contrastadas y validadas de acuerdo con el diagnóstico turistico-cultural.

El sentido último del plan es construir, sobre la base de un análisis exhaustivo de la realidad, y contando con la visión de quienes más directamente la conocen, una estrategia destinada a mejorar la situación de partida, que responda a las necesidades reales y a las percibidas como más acuciantes o importantes. Por ello, la finalidad última del trabajo es la formulación operativa de un conjunto de propuestas de actuación para la recuperación y puesta en valor del patrimonio cultural del municipio. Para cada estrategia, línea de acción y actuación concreta se propone la identificación de sus elementos constituyentes y de los que permiten hacerla operativa (Tabla 1).
La estrategia propuesta se fundamenta en 4 líneas de acción: ALOJAMIENTO, GASTRONOMÍA, NATURALEZA Y CULTURA, cuatro elementos centrales $y_{1}$ a la vez, complementarios que juntos son capaces de conformar una oferta turístico-cultural completa, dirigida a segmentos poblacionales y de mercado turistico diferenciados, y con múltiples posibilidades.

\section{Conclusiones}

La mayoría de procesos de planificación estratégica fracasan o se paralizan en la etapa de implantación. Esta afirmación, apoyada por la experiencia y la literatura especializada, hace conveniente concluir con algunas aclaraciones que deben tenerse en cuenta a la hora de formular propuestas estratégicas. En primer lugar, el sentido y la orientación de las propuestas es el de impulsar la recuperación y puesta en valor del patrimonio cultural, sabiendo que esto no siempre implica un uso turistico o la mercan-

TABLA 1. DESCRIPTORES OPERATIVOS DE LAS LÍNEAS ESTRATÉGICAS

\begin{tabular}{|c|c|c|}
\hline Elemento & Descripción & Escala \\
\hline Qué & Descripción razonada de la línea de acción o acción & Texto descriptivo \\
\hline Por qué & $\begin{array}{l}\text { Sentido de la implantación de la línea de acción o acción } \\
\text { como respuesta a una problemática u oportunidad, en el } \\
\text { marco del programa estratégico }\end{array}$ & Texto descriptivo \\
\hline Quién & $\begin{array}{l}\text { Personas o colectivos que deben liderar y/o participar en el } \\
\text { diseño e implantación de la acción o línea de acción }\end{array}$ & Texto descriptivo \\
\hline Cuándo & $\begin{array}{l}\text { Grado de prioridad en la ejecución de la acción que de- } \\
\text { penderá, a su vez, de los costes, dificultades implícitas, } \\
\text { grado de compatibilidad con otras acciones y objetivos } \\
\text { estratégicos }\end{array}$ & $\begin{array}{l}\text { De Muy Alta (5) a Muy Baja (1). Un valor cero (0) podria } \\
\text { indicar imposibilidad de realización momentánea }\end{array}$ \\
\hline Cuánto & $\begin{array}{l}\text { Costes de todo tipo derivados de la realización de la acción } \\
\text { (financieros, en recursos humanos, de instalaciones, moles- } \\
\text { tias u otros costes intangibles, etc.) }\end{array}$ & $\begin{array}{l}\text { De Muy Alto (5) a Muy Bajo (1). Un valor cero (0) podria } \\
\text { indicar imposibilidad de realización momentánea }\end{array}$ \\
\hline Dónde & $\begin{array}{l}\text { Descripción del ámbito afectado por la acción. Puede ser } \\
\text { físico (un edificio, una calle, un distrito) o temático (una } \\
\text { fiesta, una tradición, etc.) }\end{array}$ & Texto descriptivo \\
\hline Fuentes de financiación & ¿Qué posibilidades de financiación están disponibles? & $\begin{array}{l}\text { Texto descriptivo. Puede añadirse una valoración de posi- } \\
\text { bilidades de financiación disponibles de } 5 \text { (mucha cantidad } \\
\text { y/o probabilidad) y } 1 \text { (poca cantidad y/o probabilidad) }\end{array}$ \\
\hline
\end{tabular}

Fuente: Elaboración propia. 
tilización del recurso, sino también (o alternativamente) su recuperación con fines de disfrute de la sociedad local o de mejora del sentimiento de identidad con el propio territorio y la propia cultura. En segundo lugar, el ámbito territorial de la mayoría de propuestas es el término municipal de Tavernes de la Valldigna. Este hecho responde a una cuestión meramente administrativa, dado que es el propio Ayuntamiento el que encarga y financia el trabajo. Ahora bien, con frecuencia, los límites administrativos contradicen la existencia de unidades territoriales que también se refleja en el ámbito de los recursos culturales. En este sentido, es aconsejable buscar vías de colaboración supramunicipales que permiten impulsar la complementariedad entre los recursos culturales y patrimoniales de municipios vecinos. En tercer lugar, los resultados responden a un trabajo empírico de catalogación, recopilación documental y análisis exhaustivo con procedimientos objetivos. En consecuencia, las conclusiones y propuestas a las que se llega son fruto de estos análisis $y$, por tanto, derivan de ellos y en ningún caso de preferencias o gustos subjetivos de los miembros del equipo de investigación. En cuarto lugar, y en relación con el punto precedente, el análisis técnico ha sido objeto de revisión por parte de los principales colectivos sociales del municipio dentro de un proceso de participación ciudadana destinado a validar y legitimar las apreciaciones y valoraciones técnicas. Mediante este procedimiento se ha tratado de incluir el inestimable conocimiento experiencial de la sociedad local que, si bien no debe fundamentar por sí mismo posicionamientos y tomas de decisiones, debe estar presente como elemento de contraste y apoyo. Por último, la propuesta estratégica constituye un punto de referencia para la acción concertada de los agentes públicos, privados y sociales del territorio. Al tratarse de un documento estratégico, el alcance temporal de las actuaciones propuestas es diverso y va desde el momento presente en el caso de las acciones más urgentes, hasta un futuro a 10-15 años vista, en función de las prioridades y disponibilidades presupuestarias. Aunque se ha hecho un esfuerzo de concreción de los elementos que componen cada línea de acción, este Plan necesita desarrollarse, a su vez, en un Plan Director o Plan de Acción que programe con detalle cada una de las actuaciones que se decida llevar a cabo. Esta herramienta encontrará un punto de partida indispensable en el programa de acciones estratégicas.

Es frecuente encontrar documentos estratégicos consistentes en un simple listado de líneas de acción y, en todo caso, acciones concretas sin más detalle. Si bien estos documentos cumplen, desde un punto de vista formal, con el propósito de un Plan Estratégico, se vuelven poco o nada útiles al carecer de los elementos que permiten hacer operativas y llevar a la práctica las acciones propuestas. En un intento de superar esta situación, el programa estratégico propuesto contiene, para cada una de las líneas de actuación y acciones, los que, a nuestro entender, constituyen los elementos fundamentales para favorecer un adecuado proceso de implantación. De esta manera, cada una de las acciones se justifica respondiendo a las preguntas de qué es, para qué (qué problema u objeto trata de responder), quién está implicado, cómo (el modo instrumental, los procedimientos), cuándo (la prioridad que tiene en hacerse), en cuánto (las instalaciones y conocimientos necesarios, los recursos financieros y humanos), dónde (la parte del territorio al que afecta), y la financiación necesaria (fuentes de financiación potencial). De este modo, la implantación efectiva del programa estratégico no tropieza con lagunas fundamentales de información sino que queda a la voluntad de los tomadores de decisiones de aprovechar, o no, el instrumento que se pone a su disposición.

\section{BIBLIOGRAFÍA}

Case, K. E. y Fair, R. C. (1999): Principles of Economics (5th ed.), Prentice-Hall.

COM (2010): Unlocking the potential of cultural and creative industries, disponible en http://ec.europa.eu/culture/

Recibido: 17 de mayo de 2011 Aceptado: 15 de julio de 2011 our-policy-development/doc/GreenPaper_creative_industries_en.pdf.
Delgado, E. (2001): "Planificación cultural contra espacio público", en Karis, n. ${ }^{\circ} 11$, pp. 49-61, Barcelona: Fundació Interarts.

Elliot, J. (1997): Tourism: Politics, and Public Sector Management, London: Routledge.

Hall, C. M. (2000): Tourism Planning, Harlow: Prentice Hall. 
Figuerola, M. (2006): "El modelo turistico español. Déficit, retos y oportunidades", en Sáez, A.; Martín, P. y Pulido, J. I. (coords.): Estructura económica del Turismo, Madrid: Sintesis.

Moragues Cortada, D. (2006): Turismo, cultura y desarrollo, Agencia Española de Cooperación Internacional, Ministerio de Asuntos Exteriores y Cooperación. Disponible en http://www.aecid.es/ export/sites/default/web/galerias/ cooperacion/Cultural/descargas/02Turismo.CxD.pdf.

Noguera Tur, J. (2009): "Pensamiento y planificación estratégica: definición e implementación de estrategias de desarrollo", en Noguera, J. et al. (2009), Gestión y Promoción del Desarrollo Local, pp. 57-84. Serie Estudios y Documentos de la colecció Desarrollo Territorial, Instituto Interuniversitario de Desarrollo Local, Publicacions de la Universitat de València.
Nogués Pedregal, A. (2007): "From the Inside to the Inside': A New Development Model in Tourism Environments", Tourism Planning \& Development, 4: 1, 75-87, disponible en http://dx.doi. org/10.1080/14790530701311418.

Organización Mundial de Turismo (2000): Cultural Heritage and Tourism Development: A Report on the International Conference on Cultural Tourism, Siem Reap, Cambodia 11-13 December 2000 (World Tourism Organization Conference Proceedings).

Pascual, J. y Dragojevic, S. (2007): Guía para la participación ciudadana en el desarrollo de políticas culturales locales para ciudades europeas, Hanneloes Weeda_Fundacion Europea de la Cultura, 1. ${ }^{\text {a ed., p. } 70 .}$

Pearce, D. G. (1992): Tourist Organizations, Harlow: Longman Scientific and Technical.

Pike, A.; Rodríguez-Pose, J. y Tommaney, J. (2011): "Desarrollo Local y Regio- nal", Colección Desarrollo Territorial, n. ${ }^{\circ}$ 8, Publicacions de la Universitat de València.

Rausell, P. (2006): Consideraciones sobre el tránsito de Valencia hacia la ciudad global, Revista Ciudades, Valencia, Universitat de València, http://www. uv.es/cursegsm/Publiceconcult/ValenciaRevistaCiudades.pdf.

Rausell, P.; Abeledo, R.; Carrasco, J. y Martínez, P. (2007): Cultura. Estrategia para el desarrollo local, Agencia Española de Cooperación Internacional, Ministerio de Asuntos Exteriores y Cooperación. Disponible en http://www.aecid. es/export/sites/default/web/galerias/ cooperacion/Cultural/descargas/04CulturaEstrategia.pdf.

Valenzuela Rubio, M. (2009): "Ciudad y Sostenibilidad, el mayor reto urbano del siglo XXI", Lurralde Investigaciones Espaciales, n. ${ }^{\circ}$ 32, pp. 404-436.

Valls, J. F. (2004): Gestión de empresas de turismo y ocio, Ediciones Gestión 2000. 